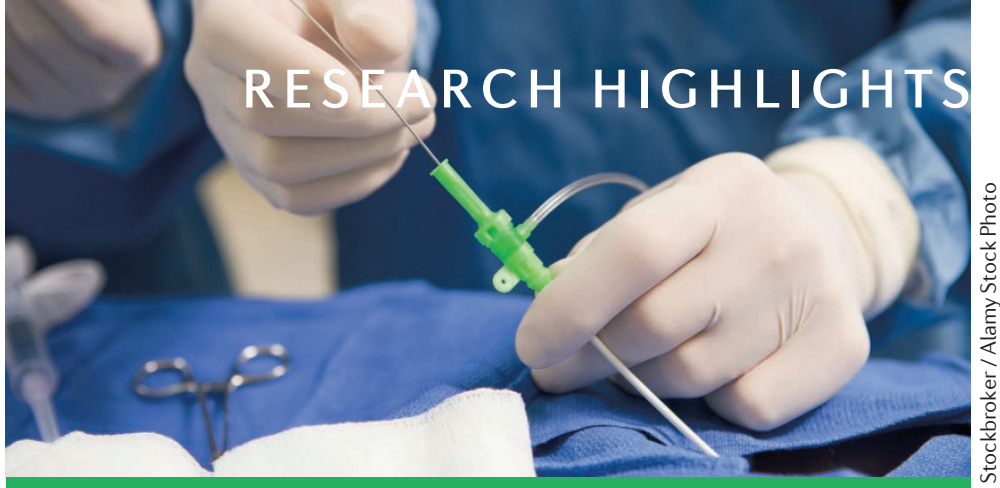

INTERVENTIONAL CARDIOLOGY

\title{
Adenosine-free assessment of stenosis severity
}

Measurement of the instantaneous wave-free ratio (iFR) is noninferior to measurement of the fractional flow reserve (FFR) for assessment of coronary artery stenosis to guide percutaneous coronary intervention. This finding was simultaneously reported by the DEFINE-FLAIR and iFR-SWEDEHEART studies, both presented at the ACC.17 Scientific Sessions and published in NEJM.

FFR-guided coronary revascularization has better patient outcomes than FFR guided by angiography only. FFR is measured by advancing a coronary pressure guidewire distal to a stenotic lesion, and then administering a vasodilator such as adenosine to assess the pressure gradient across the lesion during hyperaemia. iFR is calculated by measuring the resting pressure gradient across a coronary lesion during diastole, when microvascular resistance is low and stable. The advantage of iFR, therefore, is that the procedure does not require administration of adenosine, which can cause adverse effects.

In the DEFINE-FLAIR trial, 2,492 patients with coronary artery disease were randomly assigned to undergo iFR-guided or FFR-guided coronary revascularization. At 1 year, the primary end point (a composite of all-cause death, nonfatal myocardial infarction, or unplanned revascularization) occurred in $6.8 \%$ of the iFR group and $7.0 \%$ of the FFR group $(P<0.001$ for noninferiority). No significant differences were observed between the groups in the individual components of the primary end point. In the iFR group, only $3.1 \%$ of patients reported adverse procedural symptoms (19 with chest pain and 13 with dyspnoea), compared with $30.8 \%$ of patients who underwent FFR ( 90 with chest pain and 250 with dyspnoea). Additionally, median procedural time was significantly shorter with iFR than FFR ( 40.5 min vs $45.0 \mathrm{~min} ; P=0.001$ ).

In the iFR-SWEDEHEART study, 2,037 patients with stable angina or an acute coronary syndrome were randomly assigned to coronary revascularization guided by iFR or FFR. The primary end point (which was the same as in the DEFINE-FLAIR trial) occurred in $6.7 \%$ and $6.1 \%$ of the iFR and FFR groups, respectively ( $P=0.007$ for noninferiority). Again, the rates of the components of the primary end point did not differ significantly between the two groups. Chest discomfort during the procedure was reported by $3.0 \%$ of the patients in the iFR group and $68.3 \%$ of patients in the FFR group $(P<0.001)$.

In an editorial accompanying the study reports in NEJM, Deepak Bhatt suggests that fear of complications with the administration of adenosine, such as bradycardia, heart block, and patient discomfort from chest pain or dyspnoea, has limited the uptake of FFR. These limitations are overcome with iFR. "The use of iFR might [also] facilitate multivessel evaluation, which is viewed unfavourably by many operators because it results in a longer procedure and the need for repeat administration of adenosine," comments Bhatt. "FFR has been the evidence-based standard for invasive evaluation of [coronary stenoses of intermediate severity], but it now appears that iFR may be the new standard."

Gregory B. Lim

ORIGINAL ARTICLES Davies, J. E. et al. Use of the instantaneous wave-free ratio of fractional flow reserve in PCI. N. Engl.J. Med. http://dx.doi.org/10.1056/NEIMoa1700445 (2017)|Götberg, M. et al. Instantaneous wave-free ratio versus fractional flow reserve to guide PCI. N. Engl.J. Med. http://dx.doi.org/10.1056/NEJMoa1616540 (2017) 